\title{
SPECTROSCOPY AND DYNAMICS OF BENZONITRILE-ENZENE COMPLEXES IN A SUPERSONIC FREE-JET
}

\author{
F. LAHMANI, C. LARDEUX-DEDONDER and A. ZEHNACKER \\ Laboratoire de Photophysique Moléculaire, \\ Batiment 213-Université de Paris-Sud, 91405-ORSAY Cedex (France)
}

(Received 29 February, 1988; in final form 30 May, 1988)

\begin{abstract}
Excitation and fluorescence spectra of benzonitrile-benzene and toluene complexes seeded in helium and expanded in a supersonic free jet reveals two types of transition attributed to the coexistence of two isomeric forms. One consists of narrow bands in both excitation and emission and is characteristic of weakly perturbed van der Waals complexes. The other involves broadened absorption and gives rise to an exciplex type emission.
\end{abstract}

KEY WORDS: Spectroscopy, dynamics, benzonitrile-benzene complexes; supersonic free jet, isomers.

\section{INTRODUCTION}

In the recent years much attention has been paid to the spectroscopic study of molecular complexes involving large aromatic molecules with rare gas atoms or small molecules produced by the supersonic beam technique. ${ }^{1}$ Most of the studies were aimed to elucidate either the influence of molecular interaction (dispersive forces, hydrogen bonding) on the solvatation of aromatic molecules or to get informations on specific effects on the dynamics of excited states such as vibrational redistribution, predissociation and exciplex formation. On the other hand isolated molecular dimers of simple aromatic molecules such as tetrazine,${ }^{2}$ benzene, ${ }^{3}$ toluene, ${ }^{4}$ pyrazine ${ }^{5}$ and pyrimidine $e^{5}$ has also been the subject of extensive work as they provide model systems for condensed phase structure and relaxation phenomena. However, less information is available on aromatic mixed dimers bearing two similar chromophores close in energy as it is the case for complexes between simple substituted benzenes. These systems are interesting because they offer a way to investigate the interaction of two slightly different absorbing species which can be spectroscopically identified. We have previously undertaken an experimental and theoretical study of the jet cooled adduct between aniline and benzene. ${ }^{6}$ The present work involves a similar investigation of the benzonitrile-benzene systems. Benzonitrile is of special interest because of its large dipole moment and of the presence of two active sites of molecular interaction i.e. the benzene ring and the $C \equiv N \Pi$ electron containing substituent. A fluorescence study of benzonitrile in a supersonic free jet and its complexes with $\mathrm{Ar}, \mathrm{Kr}, \mathrm{N}_{2} \mathrm{O}, \mathrm{H}_{2} \mathrm{O}, \mathrm{CF}_{3} \mathrm{H}$ has been reported recently by Kobayashi 
and coll. ${ }^{7}$ in order to determine the role of dipole-dipole interaction in the stabilization of the complexes. We describe here the results on the electronic excitation and fluorescence spectra of benzonitrile-benzene or toluene complexes where two types of coexisting behaviour are observed, one being characteristic of a van der Waals bound system both in the ground and excited state while the other leads to an exciplex type emission.

\section{EXPERIMENTAL}

The supersonic free jet used in these experiments was formed by mixing helium (1-5 atmospheres) saturated with benzene (or toluene) and benzonitrile vapours at controlled temperature and by expanding the mixture through a $200 \mu \mathrm{m}$ nozzle. The frequency doubled output of a dye laser (coumarin $540 \mathrm{~A}$ ) pumped by the second harmonic of a Nd YAG laser (BM Industrie) was used as the excitation source. The fluorescence emission was observed 20 to 30 nozzle diameter downstream at right angle from both the molecular and laser beams and detected either directly by a photomultiplier (RTC XP 20-20 or 56 SBUVP) located behind a slit imaging the interaction zone to record fluorescence excitation spectra and fluorescence decays or through a Jobin-Yvon $60 \mathrm{~cm}$ monochromator working at $15-150 \mathrm{~cm}^{-1}$ spectral resolution in combination with a EMI-9558 photomultiplier to obtain dispersed fluorescence spectra. The fluorescence decay curves were recorded using a digital Le Croy 9400 oscilloscope. The width of the laser pulse is about $10 \mathrm{~ns}$ and the risetime of the photomultiplier is less than $3 \mathrm{~ns}$.

\section{RESULTS}

\section{Fluorescence Excitation Spectra}

The excitation spectrum of jet cooled benzonitrile ( 1 torr vapour pressure seeded in $\mathrm{He} 3$ atmospheres) recorded in the red side of the $\mathrm{O}_{0}^{0}$ transition presents several bands as noted before by Kobayashi and coll. ${ }^{7}$ The most intense at $-95 \mathrm{~cm}^{-1}$ is strongly dependent of the benzonitrile concentration and has been assigned to the dimer of benzonitrile. The other features at -113 and $-49 \mathrm{~cm}^{-1}$ are attributed to hot bands of bare benzonitrile. Upon addition of benzene a new spectrum appears in the same low energy region as shown in Figure 1 . This excitation spectrum consists of a sharp structure with the most intense band located at $-148 \mathrm{~cm}^{-1}$ from the $\mathrm{O}_{0}^{0}$ of the free benzonitrile and a "broad" congested slightly asymmetric band peaking at -78 $\mathrm{cm}^{-1}$ with a width of approximately $28 \mathrm{~cm}^{-1}$. Although the sharp structure is too complex to be fully analyzed, as several vibrational modes seem to be involved, a main vibrational progression of $20 \mathrm{~cm}^{-1}$ starting from the $\overline{\mathrm{O}}_{0}^{0}$ transition of the complex can be proposed.

Similar absorptions were found to the red side of the main vibronic bands of the monomer $\left(6 b_{0}^{1}-6 a_{0}^{1}-1_{0}^{1}-18 a_{0}^{1}\right) .^{7}$

The two systems ("sharp" and "broad" bands respectively) have the same pressure dependence as benzonitrile itself for benzonitrile partial pressure $P_{\mathrm{BN}}$ between 0.1 


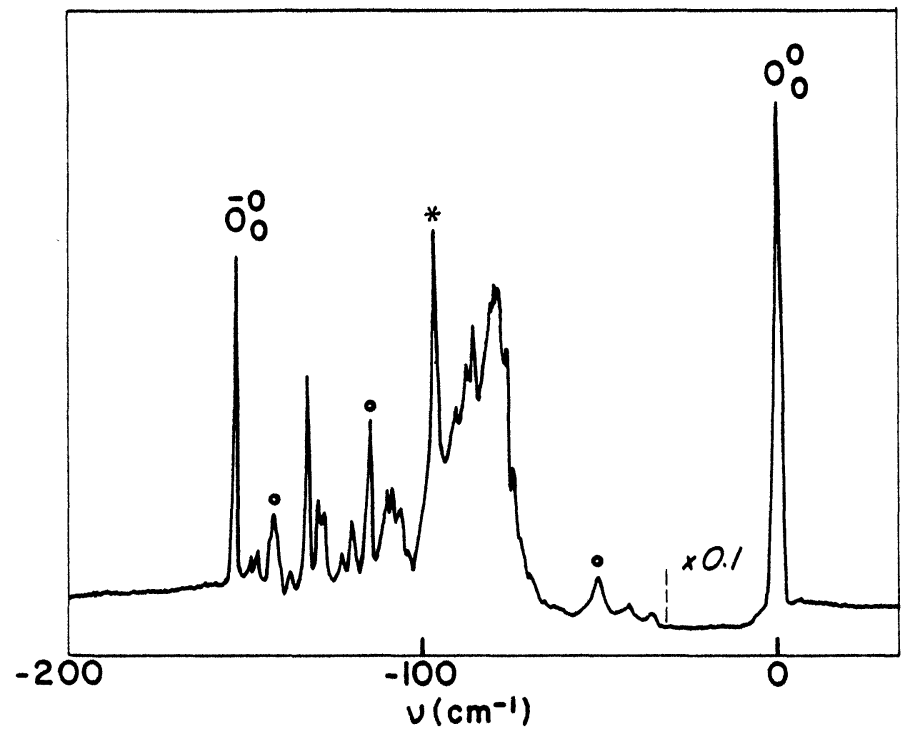

Figure 1 Part of the laser induced fluorescence excitation spectrum of the jet cooled benzonitrilebenzene van der Waals complexes in the vicinity of the $\mathrm{O}_{0}^{0}$ transition of benzonitrile. Features present in pure benzonitrile are marked by a circle $\circ$ ( hot bands) or an asterisk * (dimer).

and 0.7 torr while the benzonitrile dimer band grows as $P_{\mathrm{BN}}^{2}$. For low benzene concentrations $\left(1<P_{\mathrm{B}}<10\right.$ torr $)$ the intensity of both absorptions shows a parallel linear pressure dependence. For higher benzene concentration the "sharp" band intensity still increases with $P_{\mathrm{B}}$ while the broad band intensity starts to level off. Although the role of higher clusters such as $\mathrm{BN}_{2} \mathrm{~B}$ or $\mathrm{B}_{2} \mathrm{BN}$ where ( $\mathrm{BN}$ stands for benzonitrile and $\mathrm{B}$ for benzene) cannot be excluded, the similar pressure dependence at low partial pressure of either BN or B suggests the same 1:1 composition for the complexes responsible for sharp and broad absorptions.

Two types of excitation spectra are also observed in the case of benzonitriletoluene-complexes (Figure 2). When the emission is detected at $\lambda<300 \mathrm{~nm}$ with a solarblind photomultiplier a "sharp" structure appears with the main peak at -210 $\mathrm{cm}^{-1}$ from the $\mathrm{O}_{0}^{0}$ band of bare benzonitrile (Figure 2a). When the photomultipiler detection domain is extended over $300 \mathrm{~nm}$ a new system starting at $-163 \mathrm{~cm}^{-1}$ from the $\mathrm{O}_{0}^{0}$ band of benzonitrile and presenting a congested asymmetric structure with a sharp cut off on the high energy side is observed (Figure 2b). This latter absorption has already been observed and assigned to the mixed toluene-benzonitrile complex by Fuke and $\mathrm{Kaya}^{8}$ in experiments performed with mass selected multiphoton ionization technique. These results suggest the presence of two conformers for both benzene-benzonitrile and toluene-benzonitrile complexes.

\section{Dispersed Fluorescence Spectra and Decay Time Measurements}

Further evidence of the coexistence of two isomeric forms of the 1:1 complex of benzonitrile-benzene (or toluene) is given from the dispersed fluorescence spectra. 


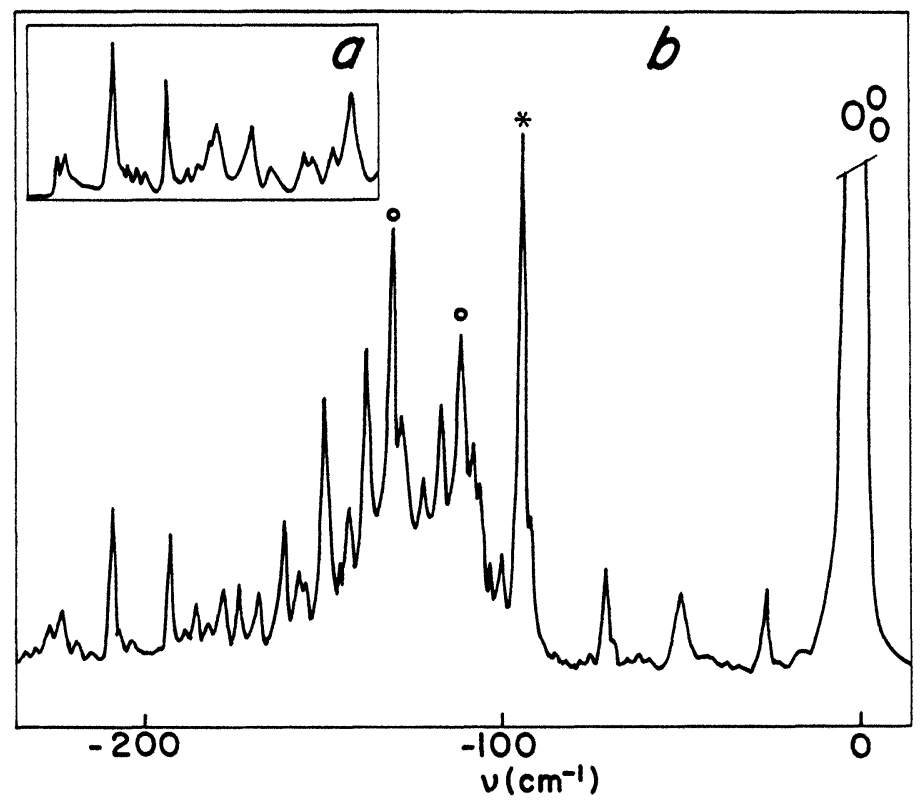

Figure 2 Part of the laser induced fluorescence excitation spectrum of the jet cooled benzonitriletoluene van der Waals complexes in the low energy region of benzonitrile $\mathrm{O}_{0}^{0}$ transition. (a) Fluorescence is detected at $\lambda<300 \mathrm{~nm}$ (solar blind 56 SB UVP RTC photomultiplier). (b) Fluorescence detection is extended toward $400 \mathrm{~nm}$ (XP 20-20 RTC photo-multiplier).

Excitation of the strong $\tilde{\mathrm{O}}_{0}^{0}$ transition of the complex leads to a structured emission similar to benzonitrile fluorescence from $\mathrm{O}_{0}^{0}$ level (Figure $3 \mathrm{a}$ ). On each vibronic band appears a sequence corresponding to the low energy intermolecular modes observed in the excitation spectrum (three bands shifted by $20 \mathrm{~cm}^{-1}$ ) (Figure 4). Fluorescence from higher members of the $\bar{v}=20 \mathrm{~cm}^{-1}$ progression observed in excitation exhibits similar structure on each vibronic band of the benzonitrile spectrum, but the Franck Condon distribution is shifted relatively to the excitation frequency: for excitation of the $\bar{v}_{0}^{1}$ band $\left(\bar{O}_{0}^{0}+20 \mathrm{~cm}^{-1}\right)$ the maximum fluorescence intensity is centered on the $\bar{v}_{1}^{1}$. In contrast with these observations, fluorescence resulting from the excitation of the "broad" feature at $-78 \mathrm{~cm}^{-1}$ is diffuse with a width of about $3500 \mathrm{~cm}^{-1}$ and is shifted towards the red relatively to resonance by about $4000 \mathrm{~cm}^{-1}$ (Figure $3 \mathrm{~b}$ ).

The fluorescence spectra presented in Figure $3 \mathrm{~b}$ shows a weak structured emission superimposed on the diffuse fluorescence. Excitation spectra recorded with the observation wavelength set either at $35,700 \mathrm{~cm}^{-1}$ or at $31,500 \mathrm{~cm}^{-1}$ allow to distinguish more clearly the two different absorptions observed previously (Figure 3). The progressions of the "sharp" system extend to the blue overlapping the "broad" absorption and leading to the weak structures observed when exciting the complex at $-78 \mathrm{~cm}^{-1}$ from the $\mathrm{O}_{0}^{0}$ of pure benzonitrile. The "broad" absorption starts at about $-120 \mathrm{~cm}^{-1}$ from the $\mathrm{O}_{0}^{0}$ transition of benzonitrile peaks at $-78 \mathrm{~cm}^{-1}$, falls off completely at $-67 \mathrm{~cm}^{-1}$ and has a halfwidth of about $28 \mathrm{~cm}^{-1}$.

The two fluorescences (structured and diffuse) differ also in their decay time as shown on Table 1. Both are shortened relative to the free benzonitrile (70 ns). For 


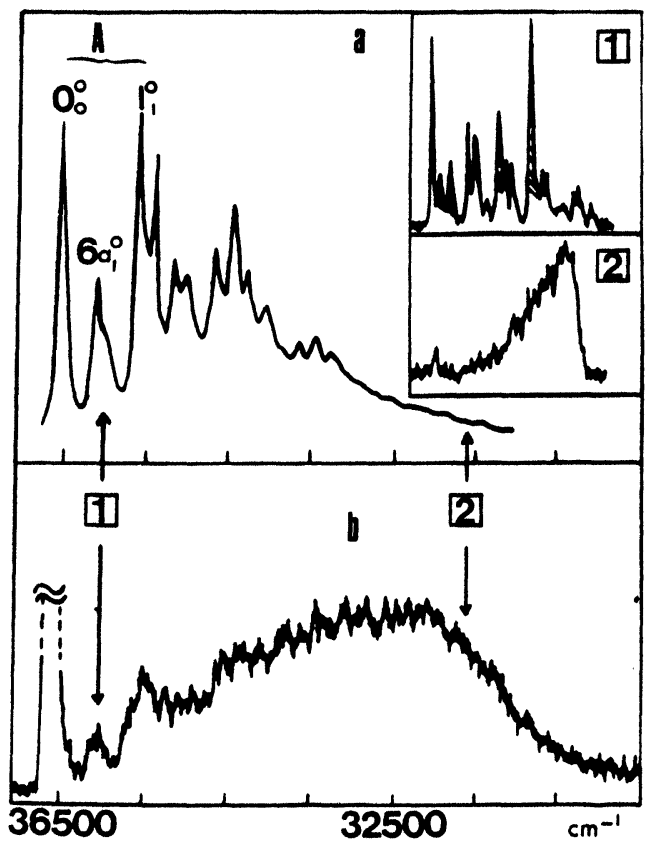

Figure 3 Dispersed fluorescence spectra of benzonitrile benzene complexes following: (a) excitation of the $O 8$ band of the complex (sharp feature at $v=v_{0}-148 \mathrm{~cm}^{-1}$ ) spectral resolution $=100 \mathrm{~cm}^{-1}$. (b) excitation of the broad feature at $v=v_{0}-78 \mathrm{~cm}^{-1}$. Spectral resolution $=100 \mathrm{~cm}^{-1}$. Insert: Excitation spectra monitored at $35,700 \mathrm{~cm}^{-1}\left(6 \mathrm{a}_{1}^{0}\right)$ (1) and $31,500 \mathrm{~cm}^{-1}(2)$.

the "sharp" features a lifetime of $\sim 40 \mathrm{~ns}$ is obtained while the "broad" red shifted emission decays with a lifetime of $\sim 30 \mathrm{~ns}$. The decay time does not depend on the excitation wavelength inside the $28 \mathrm{~cm}^{-1}$ contour of the "broad" feature correlating with the $\mathrm{O}_{0}^{0}$ transition of the free benzonitrile.

Fluorescence spectra were also obtained for the excitation of the "sharp" feature correlating with $v_{6 b}$ of bare benzonitrile $\left(v_{6 b}-155 \mathrm{~cm}^{-1}\right)$ and for the "broad" absorption $\left(v_{6 b}-83 \mathrm{~cm}^{-1}\right)$. In both cases, the emission is composed of a broad red shifted fluorescence with weak structures appearing in the region of fluorescence of benzonitrile. The fluorescence lifetimes resulting either from the "sharp" or "broad"

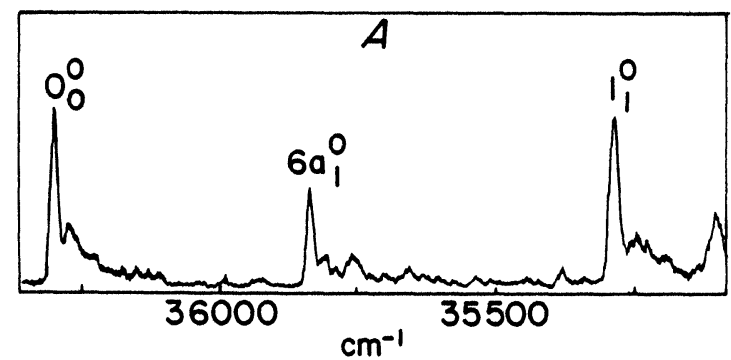

Figure 4 Extended view of the dispersed fluorescence spectra of the benzonitrile benzene complexes excited in the $O_{8}$ state (Part A of Figure 3a) showing intermolecular vibronic transitions. Spectral resolution $=20 \mathrm{~cm}^{-1}$. 
Table 1 Fluorescence lifetimes of benzonitrilebenzene excited complexes

\begin{tabular}{ll}
\hline $\begin{array}{l}v \text { excitation } \\
\text { (shift from } \mathrm{O} \text { ) } \mathrm{cm}^{-1} \text { ) }\end{array}$ & $\tau$ (ns) \\
\hline \multicolumn{1}{c}{0 (free benzonitrile) } & $70 \pm 2$ \\
-95 (dimer) & $44 \pm 2$ \\
-148 (sharp) & $43 \pm 2$ \\
-129 (sharp) & $45 \pm 2$ \\
-126 (sharp) & $40 \pm 2$ \\
-107 (sharp) & $38 \pm 2$ \\
-89 (broad) & $28 \pm 2$ \\
-80 (broad) & $28 \pm 2$ \\
+365 (sharp 6b) & $25 \pm 2$ \\
+437 (broad 6b) & $25 \pm 2$ \\
\hline
\end{tabular}

absorption bands are identical ( $25 \mathrm{~ns}$ ) and slightly shortened relative to those measured from the "broad" band correlating with the $\mathrm{O}_{0}^{0}$ transition of benzonitrile.

Similar results were obtained for the benzonitrile-toluene complexes: a van der Waals type resonant fluorescence results from the excitation of the "sharp" feature $\left(\mathrm{O}_{0}^{0}-210 \mathrm{~cm}^{-1}\right)$ while a diffuse and displaced broad emission is obtained for the excitation of the "broad" absorption.

\section{DISCUSSION}

The jet cooled-benzonitrile benzene and benzonitrile-toluene complexes present the superposition of two types of spectral features which correlate with the vibronic transitions of the benzonitrile chromophore. Energy resolved excitation spectra allow to obtain the "sharp" or the "broad" system free from the other one demonstrating clearly the presence of two different species which absorb in the same energy region but which exhibit different spectroscopic characteristics.

1) The "sharp" system observed in absorption and emission is typical of a locally excited state weakly perturbed by van der Waals interactions. The smaller red shift of the absorption $\left(150 \mathrm{~cm}^{-1}\right)$ relative to those of similar heterodimers (aniline-benzene $450 \mathrm{~cm}^{-1}$, anisole-benzene $350 \mathrm{~cm}^{-1}$ ) indicates a smaller stabilization of the excited state of the complex with respect to the ground state and may be related to the smaller change of dipole moment between ground and excited state of benzonitrile $(\Delta \mu / \mu=0.3 / 4.15)$ when compared to aniline $(\Delta \mu / \mu=0.85 / 2.38) .{ }^{9}$ The intensity distribution peaking for $\Delta v=0$ indicates a slight difference between equilibrium geometry in the ground and excited states.

2) The "broad" band observed at higher energy is indicative of a different interaction between the benzonitrile chromophore and the benzene molecule. Based on fluorescence data (diffuseness-spectral red shift), the emission can be assigned to a transition between an exciplex (upper) state and the repulsive part of the ground state. The charge transfer character of the emitting state is corroborated by the 
results of Fuke and $\mathrm{Kaya}^{8}$ where benzonitrile-toluene "broad" excitation spectrum is observed by resonance enhanced two-photon ionization technique. In this case the two-photon one-color ionization process is not allowed energetically for the locally excited complex of benzonitrile $(\mathrm{E}=4.5 \mathrm{eV})$ because of the high ionization potential of benzonitrile (IP $=9.7 \mathrm{eV}$ ). If the excited state of the complex is coupled with the charge transfer state correlating with benzonitrile ${ }^{-}$toluene $^{+}$the ionization threshold can be reduced because of the lower ionization potential of toluene (IP = $8.83 \mathrm{eV}$ ) and the two-photon one-color ionization process is then energetically possible.

These spectroscopic data and decay times suggest the coexistence of two different configurations of the mixed dimer. The excited state of one isomer decays by resonant emission as usually observed for a van der Waals complex while the other form evolves to an exciplex state from which emission takes place. The presence of two isomeric forms has been proposed in the case of perylene- $\mathrm{N}$ monomethylaniline, anthracene-dimethyl aniline ${ }^{10}$ and perylene-ammonia ${ }^{11}$ where a similar behaviour was evidenced.

The observation of the broad and displaced exciplex like fluorescence following excitation of the van der Waals complex ("sharp") correlating with the $v_{6 \mathrm{~b}}$ excitation of bare benzonitrile $\left(\mathrm{O}_{0}^{0}+517 \mathrm{~cm}^{-1}\right)$ suggests that the barrier to exciplex formation from the initially localized excited state of the van der Waals isomer is less than 517 $\mathrm{cm}^{-1}$.

Calculations on mixed dimers of benzene compounds (aniline-benzene, anisolebenzene) agree to show that a slightly displaced face geometry of both aromatic rings is the most stable configuration with a binding energy of $\sim 1300 \mathrm{~cm}^{-1}$. Although the benzonitrile-benzene complex structure has not been yet calculated, similar parallel stacked geometry can be expected in the present case and the binding energy may be even larger in the case of benzonitrile complexes as the electrostatic interaction may bring an important contribution to the stabilization of the complex due to its large dipole moment (4.14D). Our data show that two different geometric isomers are present in the jet, so we must assume two minima in the ground state potential energy surface separated by a low energy barrier. A parallel configuration of the aromatic rings with maximum overlap of the $\Pi$ electron system may be responsible for the van der Waals structured spectra while a more displaced or tilted geometry where the $\mathrm{C} \equiv \mathrm{N}$ substituent is in close interaction with benzene may preferentially couple with a charge transfer curve since this orientation favors maximum overlap between donor and acceptor orbitals.

A crude evaluation of the ionic charge transfer potential curve between benzonitrile as the electron acceptor (Electronic affinity $=0.25 \mathrm{eV}$ ) and benzene as the donor (IP $=9.25 \mathrm{eV}$ ) has been performed using the usual formula:

$$
V_{\mathrm{CT}}=\mathrm{IP}-\mathrm{EA}-e^{2} / r+\mathrm{VB}
$$

where $V_{\mathrm{B}}=\mathrm{A}_{e}^{-\lambda r}-\mathrm{C} / r^{6}$ is the Buckingham type potential similar to that developed in Ref. 6: the first term represents the repulsive interaction and the second term, the attractive dispersive term. This calculation gives a minimum for $r=3 \AA$ at 34,600 $\mathrm{cm}^{-1}$ i.e. $1900 \mathrm{~cm}^{-1}$ below the $\mathrm{O}_{0}^{0}$ transition of the free benzonitrile. ${ }^{12}$ 
If the binding energy of the locally excited state of the complex is of the same order of magnitude, as it is reasonable to assume, the charge transfer and locally excited states are near resonant. The situation is similar to the intermediate case described by Castella $e t$ al. ${ }^{10}$ and can induce strong mixing between the two isoenergetic states resulting in a broadening of the excitation spectra.

But, as seen previously in the case of aniline-benzene, the addition of an induction term $V_{\text {ind }}=-\left(\mathrm{C}_{\mathrm{BN}}{ }^{2} \alpha_{\mathrm{B}}+\mathrm{C}_{\mathrm{B}}^{2} \alpha_{\mathrm{BN}}\right) / 2 r^{4}$ where $\alpha_{\mathrm{B}}$ and $\alpha_{\mathrm{BN}}$ are the polarizabilities of the ions-which have been taken to be equal to the polarizabilities of the neutral molecules $\left(\alpha_{B}=10.32 \AA^{3}\right.$ for benzene and $\alpha_{B N}=14.5 \AA^{3}$ for benzonitrile)-would lower the charge transfer state of about $4000 \mathrm{~cm}^{-1}$.

However, this model assuming point charges centered on $\mathrm{B}^{+}$and $\mathrm{BN}^{-}$is oversimplified for large molecules and leads to an over estimation of the coulombic energy. For $\Pi \Pi$ complexes, as stated by Mulliken, ${ }^{13}$ it is more realistic to consider that the charges are distributed over the benzene and benzonitrile molecules. This leads to a coulombic energy of $3.5 \mathrm{eV}$ instead of $4.5 \mathrm{eV}$ at $3 \AA$. If this is the case, the assumption of mixing between quasi isoenergetic charge transfer (CT) and locally excited (LE) states no more holds. The observed exciplex emission should then be explained in terms of configuration interaction between locally excited states and higher energy charge transfer states as it has been proposed to account for the low energy emission of benzene excimer ${ }^{14}$ Such a scheme is proposed on Figure 5.

The asymmetric shape of the excitation band responsible for the exciplex emission, showing a sharp cut off towards the blue may be due to a steep decrease of the transition probabilities in the Franck Condon region which corresponds to the ground state equilibrium geometry of the B complex, due to a specific shape of the excited potential energy surface; a very flat surface with a low barrier (Figure 5) could account for the observed shape.

The red shift observed in the fluorescence spectrum suggests that the exciplex state

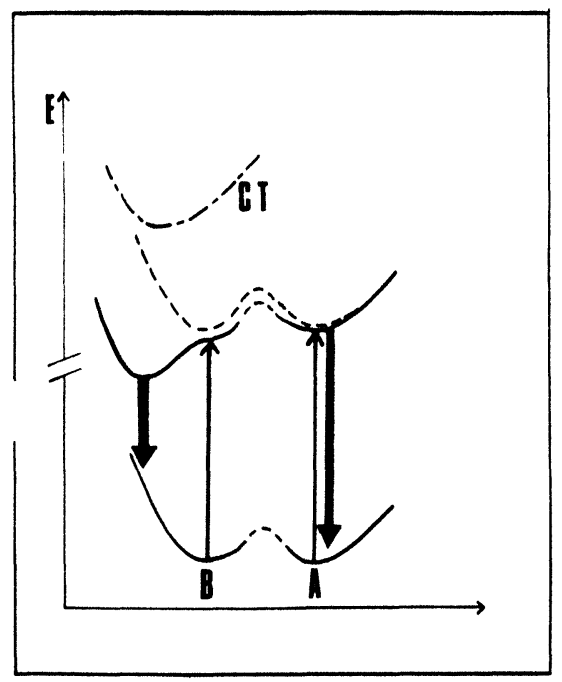

Figure 5 Schematic potential energy diagram of the benzonitrile-benzene complex along the isomerization coordinate. Dashed lines represent the non-perturbed surfaces. Arrows indicate the absorption and the fluorescence of the "sharp" A and "broad" B isomers. 
emits towards the repulsive part of the ground state potential energy surface. If this is the case, from the $4000 \mathrm{~cm}^{-1}$ red-shift, we can assume that the exciplex state lies between 1500 and $2500 \mathrm{~cm}^{-1}$ below the locally excited state. This $4000 \mathrm{~cm}^{-1} \mathrm{red}$ shift is less important than usually observed for known exciplexes, which may explain why the exciplex emission has not been reported in solution.

However, the blue cut off in the "broad" excitation spectrum could also indicate the sudden onset of a fast non-radiative channel which competes with fluorescence. But the same broad asymmetrical bands are reproduced at higher energy corresponding to higher vibronic levels of the benzonitrile chromophore, excluding thus the occurrence of an energy dependent process. A photochemical process requiring a proper orientation of the reactant, which can be induced by low frequency intermolecular modes may be responsible for this behaviour.

Another point can be noticed in the present experiments when compared with other studies on jet cooled exciplexes ${ }^{10,11}$ which is relative to the lifetime of the exciplex emission. While in most of the observed systems, the red shifted diffuse fluorescence decays with a longer lifetime than the bare chromophore (except the 1 cyanonaphthalene-triethylamine complex ${ }^{15}$ ) one observes here a shortening of the lifetime not very different from that of the van der Waals complex. Furthermore this decay time is slightly dependent of the excess energy in the excited state of the complex (Table 1). Thus the shortened lifetime is not due to the competition between fluorescence and an energy dependent non-radiative process such as intersystem crossing which rate should increase with energy. This exciplex lifetime reflects more probably the decay into a non-radiative channel which is relatively independent of the excess energy in the excited state of the complex. This process could be either the internal conversion to the ground state or a possible photochemical reaction with a low activation energy.

Although these alternatives cannot be resolved at present, it is interesting to mention, with respect to this discussion, the bimolecular photochemical reactions of benzonitrile with alkenes. Excited benzonitrile has been shown by Cantrell ${ }^{16}$ to undergo two types of cycloaddition depending on the density of electrons located on the double bond: a $2+2$ cycloaddition at the $1-2$ position of the benzene ring was found to be the major pathway for alkenes of high ionization potential (electron poor) while an addition to the $\mathrm{C} \equiv \mathrm{N}$ triple bond yielding one azetine product was the exclusive route for alkenes of low ionization potential (electron rich). The regioselectivity of the cycloaddition has been explained in terms of two exciplexes of different geometries involving two sites of complexation for the excited benzonitrile: either the benzene ring or the $C \equiv N$ part of the molecule. Further study of jet cooled adduct of benzonitrile with alkenes or conjugated dienes are under progress in order to check the present hypothesis and to identify the nature of the exciplex precursor in the photochemistry of benzonitrile with olefins or dienes.

\section{Acknowledgements}

We would like to thank D. Solgadi and C. Jouvet for their useful assistance and helpful discussions during the work. 


\section{References}

1. See for example: U. Even, A. Amirav, S. Leutwyler, M. J. Ondrechen, Z. Berkovitch-Yellin and J. Jortner, Faraday Discuss. Chem. Soc. 73, 153 (1982).

2. C. A. Haynman, D. V. Brumbaugh and D. H. Levy, J. Chem. Phys. 79, 1581 (1983).

3. (a) P. R. Langridge-Smith, D. V. Brumbaugh, C. A. Haynam and D. H. Levy, J. Phys. Chem. 85, 3472 ( 1981); (b) J. B. Hopkins, D. E. Powers and R. E. Smalley, J. Phys. Chem. 85, 3739 (1981); (c) K. H. Fung, H. L. Selzle and E. W. Schlag, J. Phys. Chem. 87, 5113 (1983).

4. K. S. Law, M. Schauer and E. R. Bernstein, J. Chem. Phys. 81, 4871 (1984).

5. J. Wanna, J. A. Menapace and E. R. Bernstein, J. Chem. Phys. 85, 777 (1986).

6. F. Lahmani, C. Lardeux-Dedonder, D. Solgadi and A. Zehnacker, Chem. Phys. 120, 215 (1988).

7. T. Kobayashi, K. Honma, O. Kajimoto and J. Tshuchiya, J. Chem. Phys. 86, 1111 and 1118 (1987).

8. K. Fuke and K. Kaya, Chem. Phys. Letters 91, 311 (1982).

9. J. L. Murov, Handbook of Photochemistry p. 153, M. Dekker Inc. NY, 1973.

10. M. Castella, A. Tramer and F. Piuzzi, Chem. Phys. Letters 129, 105 and 112 (1986).

11. O. Anner and Y. Haas, J. Phys. Chem. 90, 3298 (1986).

12. D. Solgadi, to be published.

13. R. S. Mulliken, W. B. Person in Molecular Complexes. Wiley Interscience Publ. 1969 p. 120.

14. M. T. Vaia, I. H. Hillier, S. A. Rice and J. Jortner, J. Chem. Phys. 44, 23 (1966).

15. H. Saigusa and M. Itoh, J. Chem. Phys. 86, 2588 (1987).

16. T. S. Cantrell, J. Org. Chem. 42, 4238 (1977). 